\title{
The crystal structure and cytotoxicity of centrosymmetric copper(II) complex derived from S-methyldithiocarbazate with isatin.
}

\begin{abstract}
Copper(II) complex of S-methyldithiocarbazate with isatin has been prepared and screened for their cytotoxic activities against MCF-7 (Human non-metastatic mammary gland adenocarcinoma cell line) and MDA-MB-231 (Human metastatic mammary gland adenocarcinoma cell line). The compound crystallized in an orthorhombic crystal system with a space group $\mathrm{C} 2 \mathrm{cb}$ and was found to be selectively active against MCF-7 cell line (Human non-metastatic mammary gland adenocarcinoma cell line with an IC50 value $0.45 \mu \mathrm{g} / \mathrm{mL}$. The crystal structure of this centrosymmetric Cu(SMISA)2 complex (SMISA = Schiff base formed by condensation reaction of S-methyldithiocarbazate with isatin) showed that the copper atom has a distorted square-planar geometry with the Schiff base coordinated to the metal ion as a uninegatively charged bidentate ligand through the azomethine nitrogen and thiolate sulfur.
\end{abstract}

Keyword: Copper(II); Centrosymmetric; Dithiocarbazate; MCF-7; Schiff base. 